\title{
Research on CS-MRI Reconstruction with WHT as Sparsifying Transform
}

\author{
G. Shrividya, S.H. Bharathi
}

\begin{abstract}
In this paper an efficient method for the reconstruction of Magnetic Resonance Image (MRI) from the compressively sampled MR $k$-space. Compressive Sensing (CS) gives an efficient structure for getting back the signal or image from lesser measurements than that are really necessary according to the Nyquist criterion. The Walsh Hadamard transform is used as the sparsifying transform. In the proposed work radial and Cartesian sampling patterns are applied on $k$-space to collect minimum samples and MR image is recovered by taking Inverse Fourier transform of the $k$-space data. The Qualitative and quantitative analysis of the reconstructed images depict that the performance of Walsh Hadamard Transform as sparsifying transform gives better result in comparison with DFT. Experiments conducted on the MR Images of brain and knee show that proposed method gene-rates good quality images.
\end{abstract}

Keywords--- CS-MRI, MR Reconstruction, Compressed Sampling, Undersampling, Sampling Trajectory, Wlash Hadamard Transform, Sparsifying Transform.

\section{INTRODUCTION}

Magnetic Resonance Imaging is a non-invasive imaging scheme that generates images of internal organs of the human body. It is an effective non-invasive imaging modality used in the diagnosis of wide range of diseases [1]. Excellent soft tissue contrast of MRI enables good visualization of tissues and organs within the patient body. MRI works on the principle of Nuclear Magnetic Resonance (NMR). During data acquisition phase, the echo signals are stored in kspace. The collected crude k-space data is transformed into an image by suitable reconstruction method which is then visualized by medical practitioners for diagnostic and analysis purposes. Main drawback of MRI is that it is inherently a slow process [2]. This is because the $\mathrm{k}$-space samples are acquired sequentially in time. Therefore various methods are developed to reduce the scan time by collecting lesser number of samples and to reconstruct the image using minimum number of samples. This process speeds up the scanning process.

The traditional approach of reconstructing signals or images from measured data follows the well-known ShannonNyquist's sampling theorem, which states that the sampling rate must be twice the highest frequency. Similarly, the fundamental theorem of linear algebra suggests that the number of collected samples (measurements) of a discrete finitedimensional signal should be at least as large as its length (its dimension) in order to ensure reconstruction.

The idea of CS, which was developed in recent years by, Donoho [3],Candes [4] and Lustig et al., [5] applied it on MRI. In recent times, applying the theory of Compressive Sensing or compressive sampling in MR imaging is of great

Revised Version Manuscript Received on August 14, 2019.

G. Shrividya, Research Scholar, School of E\&C Engineering, REVA University \& Faculty, Dept. of E \& C, NMAMIT, Nitte, India.

S.H. Bharathi, School of E\&C Engineering, REVA University, Bengaluru. interest. Compressive sampling reduces the data collection time which in turn reduces patient's exposure to magnetic field [5]. In traditional sampling methods the images are sampled at Nyquist's sampling rate which is twice the largest frequency present in the signal of interest [4]. CS-MRI enables superior quality image reconstruction from undersampled $\mathrm{k}$-space data. It is done by evaluating constrained minimization difficulties by means of nonlinear optimization techniques by utilizing the sparsity of images in a particular sparsifying transform. Using this strategy it is possible to reconstruct MRI from reduced measurements, thus minimize the imaging time.

CS technique makes use of sparse nature of the signal in some domain and performs the reconstruction of complete signal / image from comparatively a small number of samples. Due to the high correlated feature of MRI data, the image reconstruction from partial $k$-space using CS approach will have an effect on the time taken for MRI of patient[15]. In MRI k-space, energy distribution of image information is concentrated more at central region than at peripheral region. Since energy allocation is non uniform, a random undersampling technique will result in low frequency aliasing. Therefore variable density undersampling pattern is used which selects more samples from the central region of k-space and lesser samples from the outer peripheral region [7]. Such optimum sampling pattern should consider the energy distribution of k-space [14]. An adaptive learning sparsifying transform appraoch(dictionary based) is also used for MRI reconstrcution from higly undersampled MR k-space[13]. In this paper we demonstrate that the Walsh Hadamard transform can also be used as the sparsifying transform in CS MRI. The proposed work is compared with the standard DFT technique. Total Variation is also used as a penalty to suppress noise in the reconstruction. Performance analysis of CS-MRI reconstruction for different sampling percentages is carried out in terms of PSNR, MSE and SSIM. The efficiency of the proposed method is established in the quality of reconstructed MR image.

\section{METHODOLOGY}

\subsection{Proposed method}

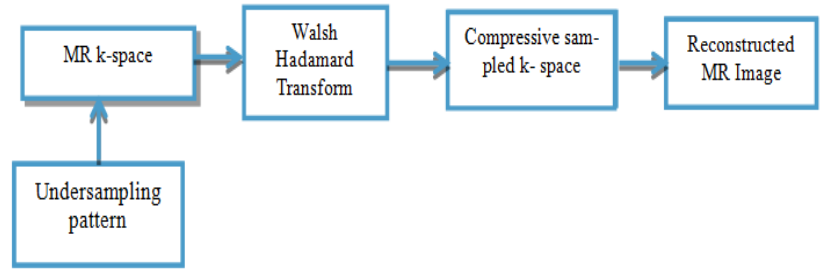

Figure 1: Block diagram of the proposed method 
The block diagram of the proposed work is as shown in Figure.1. The undersampling pattern is applied on the generated k-space. The Walsh Hadamard Transform (WHT) is used as sparsifying transform. The Cartesian or Radial sampling patterns are used to collect samples from the k-space. The sampling patterns used for compressive sampling are as shown in Figure.2. The MR image is reconstructed using the reconstruction standard algorithm. The performance of the WHT as sparsifying transform is compared with the standard DFT.

\subsection{MR k-space}

The raw MR data collected from the scanning machine is stored in k-space. The raw data is in spatial domain which will be subjected to Fourier transform to generate the MR image. Each slice contain information about the frequency, phase and intensity of the signal. The final image is reconstructed by applying Inverse Fourier Transform on the kspace. The central region of k-space holdcontrast information whereas the peripheral region contains spatial resolution[9]. The sampling pattern is designed in such a way to collect more samples from the central region than the peripheral. Hence the quality of reconstruction will not suffer even with lesser number of $\mathrm{k}$-space samples. Undersampling the $\mathrm{k}$-space will reduce the time consumed for imaging thereby minimizing the discomfort caused due to long scanning time.

\subsection{Compressive Sampling of the $k$-space}

CS takes the advantage of sparsity or compressibility of real world signals. Natural signals have concise characterization when expressed using proper basis $\psi$. Here $\boldsymbol{\psi}$ denotes the linear operator responsible for the transformation of the data from pixel representation to any other selected representation. Incoherence deals with the idea about duality of the signal in time and frequency domain. This leads to faster measurements with lesser memory requirement. Undersampling patterns are developed in such a way to preserve more information at the center than at the periphery of the k-space since majority of the information in k-space image resides at the center. Sparsity and Incoherence are the two conditions to be satisfied to apply CS on MRI.

For a good Signal to Noise Ratio (SNR) and a smaller Mean Squared Error (MSE) more samples must be collected near the origin of the k-space. The variable-density sampling scheme targets to collect larger number of samples near the center of the k-space[10]. Figure. 2 shows the Cartesian pattern for heavy sampling of central k-space lines and lesser selecting lines near the edges.
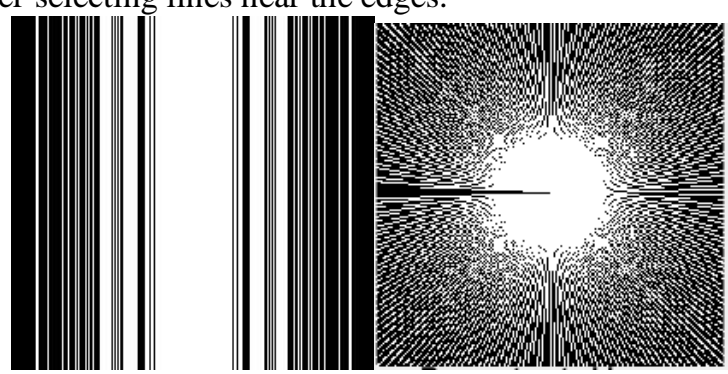

(a) (b)

Figure 2: (a) Cartesian undersampling pattern (b) Radial Undersampling Pattern
The significant information from the k-space is acquired by applying the sampling patterns as shown in Figure.2(a) and (b). The undersampled data obtained from Cartesian sampling as well as radial sampling pattern[8] are used for the reconstruction of MR image. The white pixels in the sampling pattern correspond to the locations from where the samples are collected. The sampling patterns shown in Figure. 2(a) and (b) collect more samples near the center of kspace (the density of white lines is more) and less samples collected from the peripheral regions(black lines are more).The number of $\mathrm{k}$-space lines that are need to be undersampled from the fully acquired k-space data is known as sampling percentage [14]. The quality of reconstruction is analyzed for lesser sampling percentages.

\subsection{Walsh Hadamard Transform}

Walsh Hadamard Transform (WHT) is a fast transformation method which makes use of only subtraction and addition operations. The energy compaction characteristics in a WHT are low. Pattern matching can be efficiently performed in WHT domain. In signal processing application WHT is non-sinusoidal, orthogonal transformation technique that splits a signal into a basis function set. These decomposed basis functions are called as Walsh functions and these functions are rectangular or square waves which has the values of +1 or -1 . WHT are also referred as Hadamard (Walsh, or Walsh-Fourier transforms)[11]. WHT makes use of basis functions and provides piece-wise constant discrete image band limited approximation.

$$
\begin{gathered}
Y_{n}=\frac{1}{N} \sum_{i=0}^{N-1} x_{i} W A L(n . i), n=1,2, \ldots, N-1(1) \\
x_{i}=\sum_{n=0}^{N-1} y_{n} W A L(n, i), i=1,2, \ldots, N-1(2)
\end{gathered}
$$

where $i=1,2, \ldots, N-1$ and $W A L(n . i)$ are Walsh functions, $\mathrm{N}$ is the scaling factor. The Walsh matrix is symmetric. The forward and inverse transformations are equal operation except for the scaling factor of $1 / \mathrm{N}$. The equation (1) represents the Forward WHT Transform and the equation (2) represents the Inverse WHT respectively.

\subsection{CS Reconstruction}

The general form of data acquirement with incomplete samples for CS-MRI is given as:

$$
b=R x(3)
$$

where $x$ is the reconstructed image, $b$ is the acquired kspace measurement and $R$ is the undersampled Fourier Transform operator which directly relies on the k-space undersampling scheme. Let $\Psi$ denote the linear operator that transforms the data from pixel depiction into the selected depiction. $x$ can be correctly reconstructed from portion of $\mathrm{k}$ space by evaluating the minimization problem which is given as[5]:

$$
\text { minimize }\|\Psi x\|_{1} \text { s.t. }\|R x-b\|_{2}<\epsilon(4)
$$

where $\epsilon$ is a statistic that describes the magnitude of the error defined as the noise variance or the maximum allowable error in the approximation. Here the $l_{1}$ norm $\|x\|_{1}=$ $\sum_{i}\left|x_{i}\right|$. Minimizing the objective function $\|\Psi x\|_{1}$ promotes the sparsity of the images. 


\subsection{Total Variation Minimization}

Reconstruction from sub-Nyquist acquisition results in artifacts which are due to undersampling of high spatial frequencies. This can be resolved by smoothing filters. But smoothing results in blurring of image due to the loss of edge details.

To generate the images with suitable quality from subNyquist acquisition a technique called Total Variation (TV) minimization can be used which is done by minimizing the pixel by pixel finite differences in both horizontal and vertical directions across an image. Total Variation minimization procedure is used to improve the quality of the reconstructed image[12].

The non-linear convex program is used to reconstruct the MR image using the down sampled k-space is applied along with TV minimization as given in equation(3) below:

minimize $\left\{\|\Psi x\|_{1}+\alpha . T V(x)\right\}$ such that $\left\|F_{u} x-y\right\|_{2}<$ $\varepsilon(5)$

where $x$ is the reconstructed image, $y$ is the deliberate $\mathrm{k}$ space data, $F_{u}$ is the under sampled Fourier transform, $\Psi$ is the sparsifying transform, $T V($.$) is the total-variation and$ $\varepsilon$ controls the fidelity of their construction, $\alpha$ is the regularization parameter. The equation (5) finds the sparsest of every single solution to the underdetermined linear system by limiting the $l_{1}$-norm of the measured data. The threshold parameter $\varepsilon$ is usually set below the expected noise.

\section{EXPERIMENTAL RESULTS AND DISCUS- SIONS}

CS provides an efficient way to acquire and reconstruct natural images from a limited number of linear projection
Cartesian DFT

$80 \%$

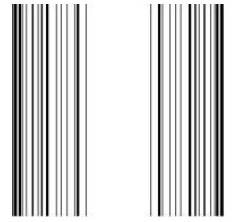

$60 \%$

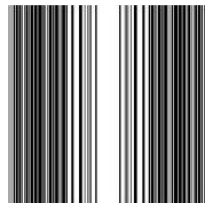

$40 \%$

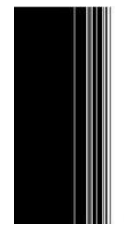

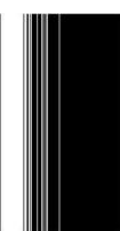

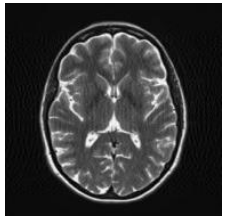

WHT Radial
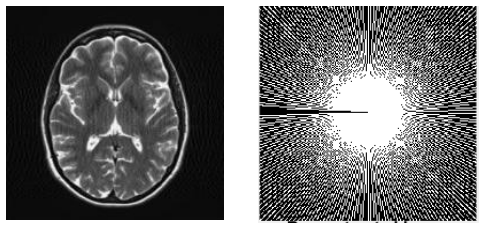

DFT

WHT

Figure 3: (a) Original MR Brain Image (b) k-space

Figure 3(a) shows the original MR image of brain.The kspace generated is shown in Figure 3(b). The sampling pattern is masked with the k-space of input MR image to compressively sample the k-space. Thereby only few important samples are acquired.

The reconstruction of the MR image from k-space is performed by taking IDFT of the k-space samples. Since the kspace is undersampled nonlinear optimization technique is used to reconstruct the image.
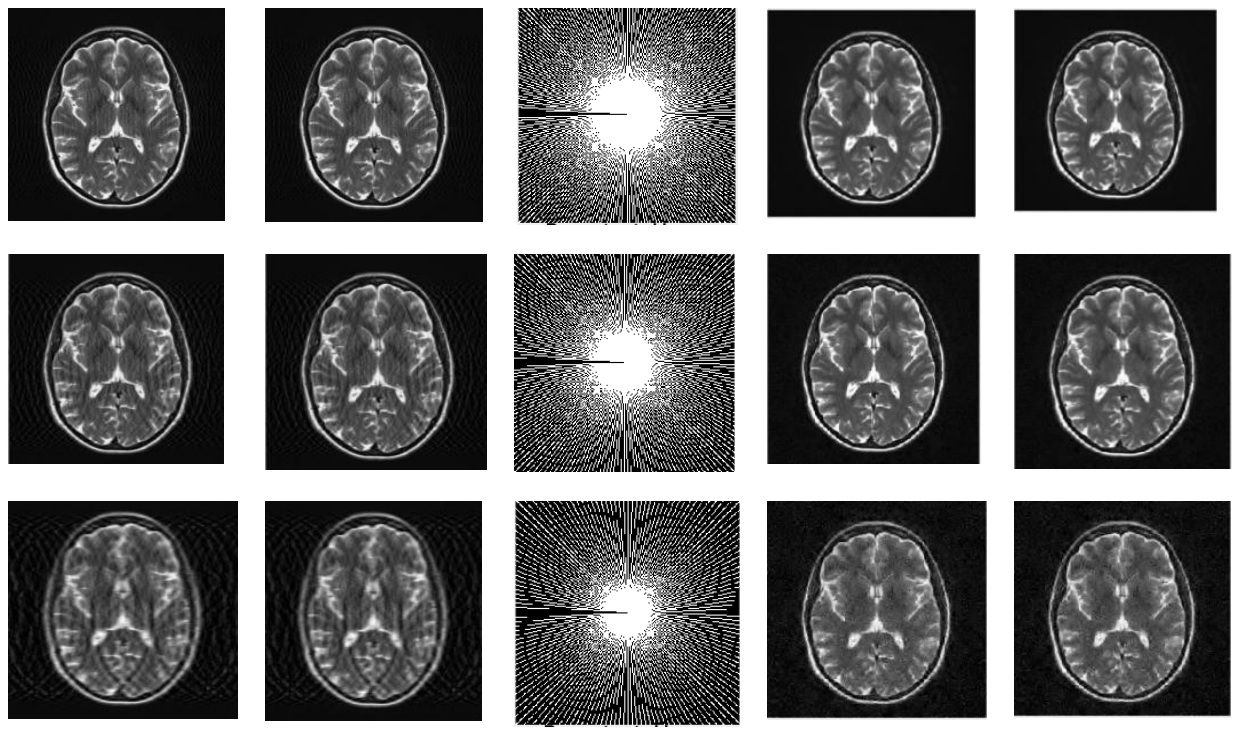

Figure 4: MR Image Reconstructed using DFT and

WHT for Cartesian and Radial Sampling Pattern

shown in Figure 4. As the sampling percentage reduces reconstruction suffers from artifacts in the reconstructed im-

The under-sampled data obtained from Cartesian sampling pattern are used for the reconstruction process using DFT and WHT technique. The simulation results are as age. To reduce the artifacts total variation minimization technique is applied.

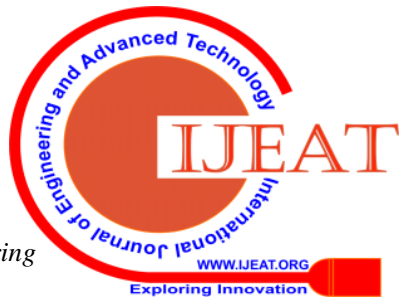


Table I: Numerical analysis of MR image Reconstruction using WHT as sparsifying transform

\begin{tabular}{|c|c|c|c|c|c|c|c|}
\hline \multirow[b]{2}{*}{ MRI } & \multicolumn{4}{|c|}{ Cartesian sampling without TV } & \multicolumn{3}{|c|}{ Cartesian sampling with TV } \\
\hline & $\begin{array}{c}\text { Sampling } \\
\text { Percentages }\end{array}$ & PSNR & MSE & SSIM & PSNR & MSE & SSIM \\
\hline \multirow{5}{*}{ Brain of patient 1} & 80 & 45.38 & 1.88 & 0.9893 & 45.91 & 1.66 & 0.9914 \\
\hline & 70 & 43.16 & 3.14 & 0.9846 & 43.65 & 2.80 & 0.9876 \\
\hline & 60 & 40.18 & 6.23 & 0.9716 & 40.75 & 5.46 & 0.9781 \\
\hline & 50 & 35.71 & 17.42 & 0.9262 & 36.36 & 15.01 & 0.9462 \\
\hline & 40 & 31.61 & 44.8 & 0.8449 & 32.29 & 38.29 & 0.8873 \\
\hline \multirow[t]{5}{*}{ Knee } & 80 & 40.17 & 6.24 & 0.9616 & 40.67 & 5.57 & 0.9727 \\
\hline & 70 & 38.20 & 9.82 & 0.9447 & 38.62 & 8.93 & 0.9597 \\
\hline & 60 & 36.29 & 15.26 & 0.9183 & 36.69 & 13.92 & 0.9402 \\
\hline & 50 & 34.06 & 25.48 & 0.8714 & 34.48 & 23.17 & 0.9081 \\
\hline & 40 & 32.02 & 40.76 & 0.8120 & 32.45 & 36.95 & 0.8651 \\
\hline \multirow{5}{*}{ Brain of patient 2} & 80 & 45.88 & 1.67 & 0.9878 & 43.36 & 1.50 & 0.9902 \\
\hline & 70 & 43.99 & 2.59 & 0.9835 & 44.46 & 2.32 & 0.9867 \\
\hline & 60 & 41.58 & 4.51 & 0.9742 & 42.11 & 4.0 & 0.9801 \\
\hline & 50 & 38.19 & 9.85 & 0.9443 & 38.82 & 8.5 & 0.9594 \\
\hline & 40 & 34.92 & 20.90 & 0.8920 & 35.62 & 17.80 & 0.9220 \\
\hline
\end{tabular}

The numerical analysis carried on the set of data which consist of MR data of brain of patient 1 and brain MRI data for patient 2 and knee MR data. Table I. displays the PSNR, MSE and SSIM measured on the reconstructed MR Image when Cartesian undersampling pattern is applied on the kspace to collect samples and WHT is used as sparsifying transform. With the sampling percentage of 40, appreciable values generated for PSNR, MSE and SSIM. SSIM at $40 \%$ sampling percentage is 0.8873 for brain image of patient 1 , 0.8651 for knee and 0.9220 for brain image of patient 2 . This means the quality of the reconstructed image is almost similar to the image reconstructed using maximum number of samples. Total variation minimization reduces the noise in the reconstruction, improving the quality of reconstructed image.

Table II: Numerical analysis of MR image Reconstruction using WHT as sparsifying transform

\begin{tabular}{|c|c|c|c|c|c|c|c|}
\hline \multirow[b]{2}{*}{ MRI } & \multicolumn{4}{|c|}{ Radial sampling without TV } & \multicolumn{3}{|c|}{ Radial sampling with TV } \\
\hline & $\begin{array}{c}\text { Sampling } \\
\text { Percentages }\end{array}$ & PSNR & MSE & SSIM & PSNR & MSE & SSIM \\
\hline \multirow[t]{5}{*}{ Brain of patient 1} & 80 & 44.32 & 2.40 & 0.9874 & 44.82 & 2.41 & 0.9899 \\
\hline & 70 & 41.52 & 4.57 & 0.9788 & 42.04 & 4.06 & 0.9832 \\
\hline & 60 & 37.62 & 11.24 & 0.9504 & 38.25 & 9.72 & 0.9630 \\
\hline & 50 & 33.12 & 31.68 & 0.8789 & 33.81 & 27.04 & 0.9125 \\
\hline & 40 & 28.85 & 84.70 & 0.7633 & 29.53 & 72.39 & 0.8273 \\
\hline \multirow[t]{5}{*}{ Knee } & 80 & 39.01 & 8.15 & 0.9528 & 39.45 & 7.37 & 0.9657 \\
\hline & 70 & 36.97 & 13.06 & 0.9292 & 37.36 & 11.92 & 0.9479 \\
\hline & 60 & 34.96 & 20.71 & 0.8917 & 35.37 & 18.86 & 0.9218 \\
\hline & 50 & 32.83 & 33.85 & 0.8380 & 33.24 & 30.77 & 0.8830 \\
\hline & 40 & 30.70 & 55.29 & 0.7679 & 31.12 & 50.15 & 0.8332 \\
\hline \multirow{5}{*}{ Brain of patient 2} & 80 & 44.76 & 2.16 & 0.9859 & 45.24 & 1.94 & 0.9884 \\
\hline & 70 & 42.36 & 3.77 & 0.9775 & 42.89 & 3.33 & 0.9824 \\
\hline & 60 & 39.73 & 6.9 & 0.9603 & 40.32 & 6.03 & 0.9702 \\
\hline & 50 & 36.27 & 15.34 & 0.9185 & 36.93 & 13.16 & 0.9407 \\
\hline & 40 & 32.17 & 39.39 & 0.8188 & 32.90 & 33.29 & 0.8695 \\
\hline
\end{tabular}

Table II gives the numerical analysis for the application of proposed method on the same set of MR images with the radial sampling pattern is applied on the k-space to collect undersampled data and WHT is used as sparsifying transform. Qualitative and quantitative analysis on the proposed method reveals that the radial sampling pattern is inferior to the Cartesian sampling pattern. The PSNR, MSE and SSIM values for the MR image reconstructed using $40 \%$ samples of the k-space show that the k-space sparsified using WHT and radial sampling even with the combination of TV minimization performs poor compared to the former method.

For different set of MR data reconstructed image suffers from poor values of PSNR, MSE and SSIM for smaller sampling percentages with radial sampling pattern. Image quality improves by combining TV minimization with WHT.

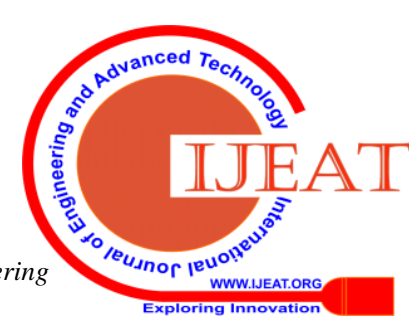


Table III: Comparative results of MR image Reconstruction using DFT TV and WHT TV

\begin{tabular}{|c|c|c|c|c|c|c|c|}
\hline \multirow[t]{2}{*}{ MRI } & \multicolumn{4}{|c|}{ DFT TV } & \multicolumn{3}{|c|}{ WHT TV } \\
\hline & $\begin{array}{c}\text { Sampling } \\
\text { Percentages }\end{array}$ & PSNR & MSE & SSIM & PSNR & MSE & SSIM \\
\hline \multirow[t]{5}{*}{ Brain of patient 1} & 80 & 42.18 & 10.96 & 0.9828 & 45.91 & 1.66 & 0.9914 \\
\hline & 70 & 40.29 & 12.46 & 0.9810 & 43.65 & 2.80 & 0.9876 \\
\hline & 60 & 36.70 & 14.01 & 0.9803 & 40.75 & 5.46 & 0.9781 \\
\hline & 50 & 34.46 & 23.42 & 0.9791 & 36.36 & 15.01 & 0.9462 \\
\hline & 40 & 32.12 & 24.19 & 0.9677 & 32.29 & 38.29 & 0.8873 \\
\hline \multirow[t]{5}{*}{ Knee } & 80 & 43.03 & 4.87 & 0.9854 & 40.67 & 5.57 & 0.9727 \\
\hline & 70 & 40.25 & 6.52 & 0.9823 & 38.62 & 8.93 & 0.9597 \\
\hline & 60 & 38.68 & 8.88 & 0.9739 & 36.69 & 13.92 & 0.9402 \\
\hline & 50 & 35.45 & 18.68 & 0.9668 & 34.48 & 23.17 & 0.9081 \\
\hline & 40 & 37.14 & 12.65 & 0.9667 & 32.45 & 36.95 & 0.8651 \\
\hline \multirow[t]{5}{*}{ Brain of patient 2} & 80 & 47.01 & 1.25 & 0.9889 & 43.36 & 1.50 & 0.9902 \\
\hline & 70 & 46.84 & 1.32 & 0.9873 & 44.46 & 2.32 & 0.9867 \\
\hline & 60 & 46.58 & 1.44 & 0.9830 & 42.11 & 4.0 & 0.9801 \\
\hline & 50 & 44.63 & 2.26 & 0.9820 & 38.82 & 8.5 & 0.9594 \\
\hline & 40 & 39.29 & 7.71 & 0.9755 & 35.62 & 17.80 & 0.9220 \\
\hline
\end{tabular}

The proposed work is compared with the standard method used for reconstruction. The numerical analysis performed on the DFT TV and WHT TV methods are given in Table III. As the sampling percentage reduces the image quality in terms of SSIM remains still near 1(standard SSIM value between 0 to 1 ). Therefore visual quality of reconstructed image is good even at lesser sampling percentages. PSNR decreases slightly with no effect on reconstruction quality. The values obtained in this experiment for different MR images depict that WHT combined with TV performs well compared to DFT technique. Further it can be observed that application of Cartesian sampling mask for k-space data collection results in better MR image than by using radial sampling pattern.
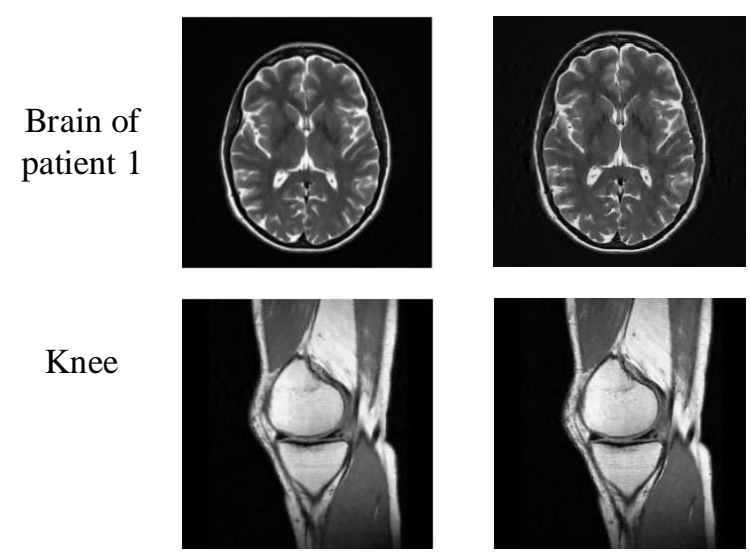

Knee

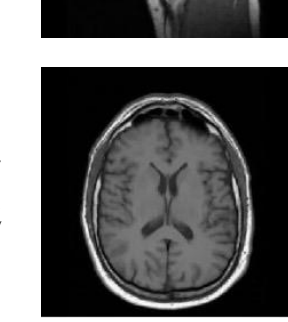

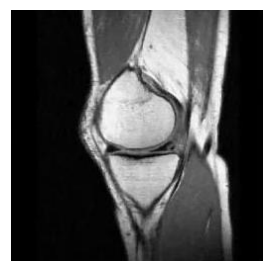

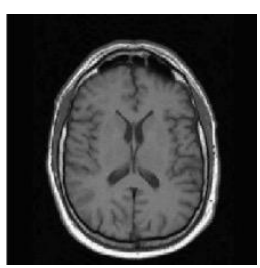

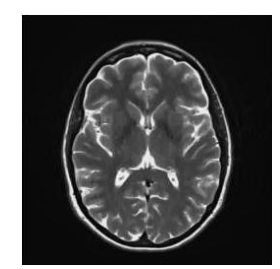
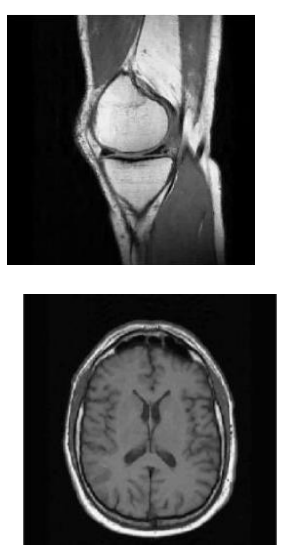
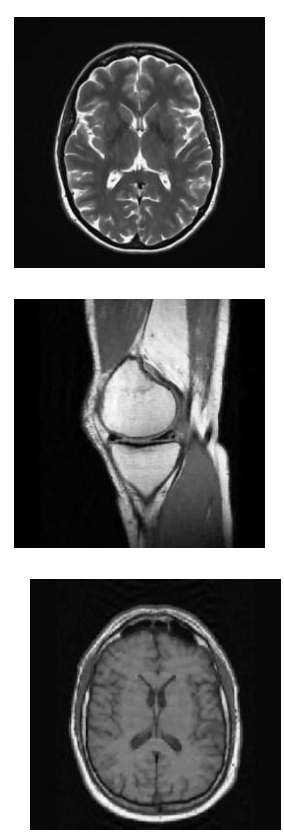
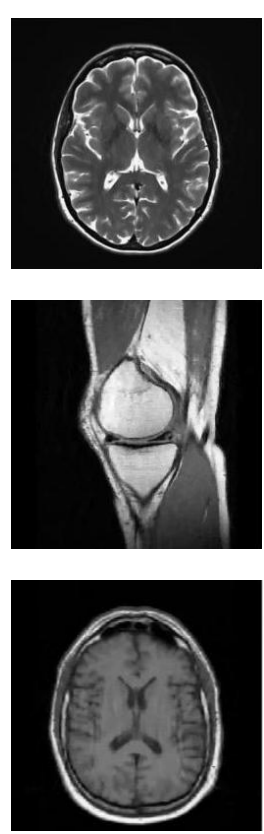

Figure 5: The reconstructed MRI of brain of patient1, knee, brain of patient 2 using Cartesian sampling pattern for DFT for different scan percentage with TV for $80,70,60,50,40$ percent sampling respectively

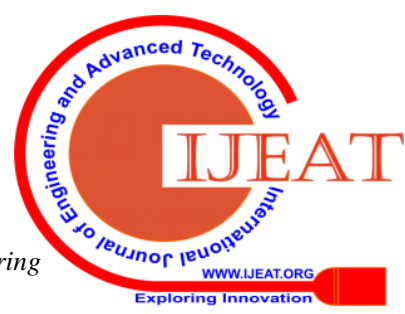



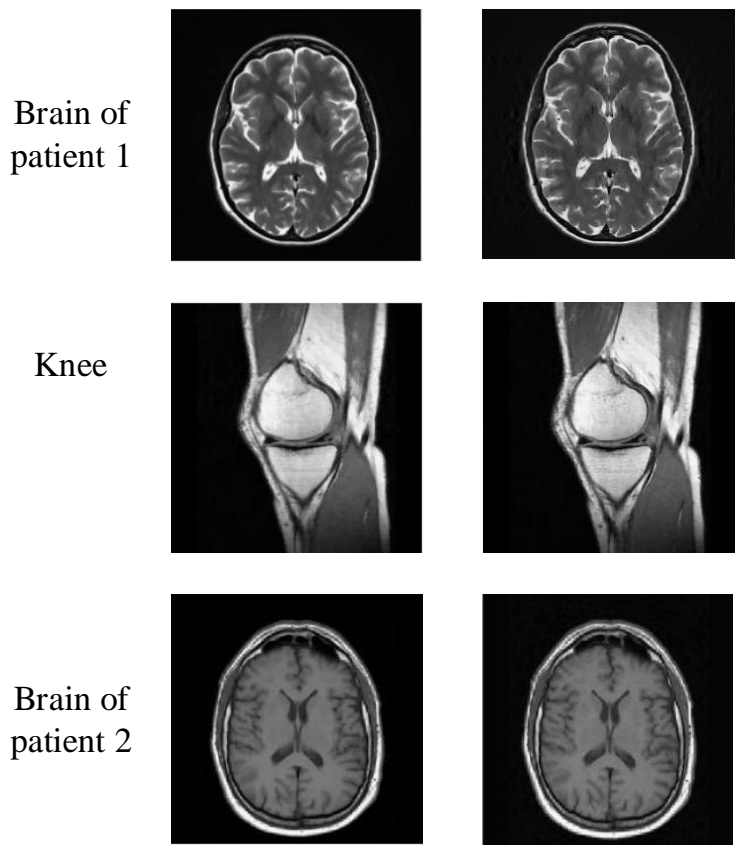
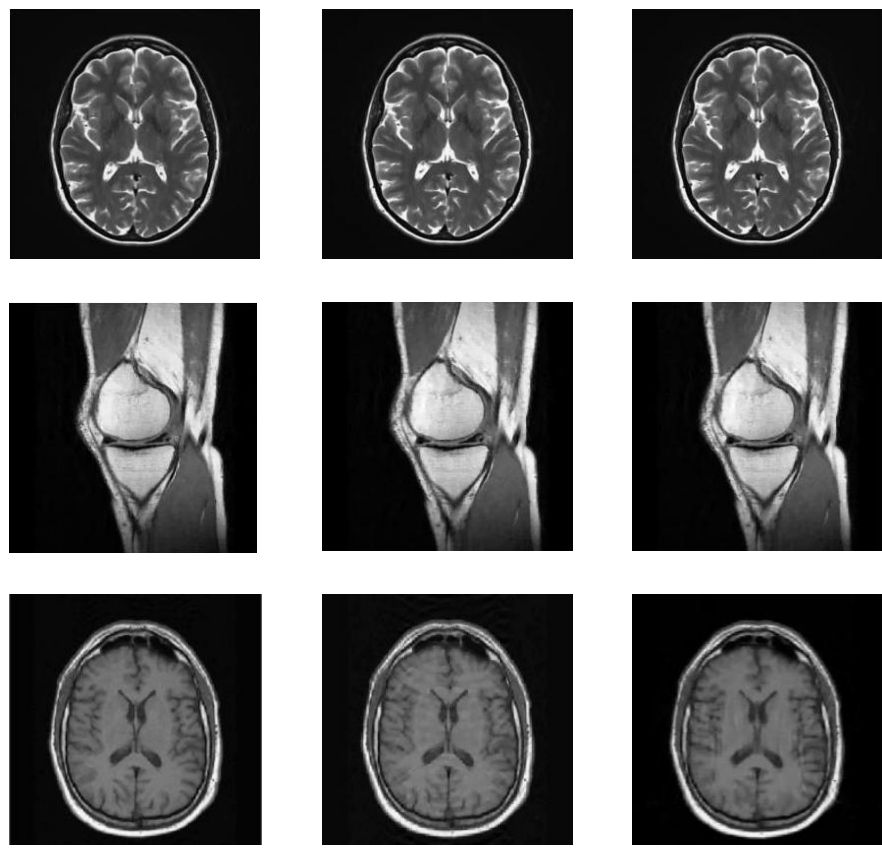
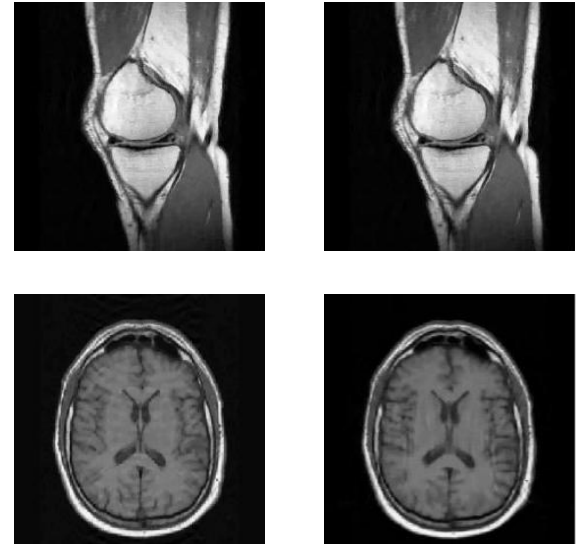

Figure 6: The reconstructed MRI of brain of patient1, knee, brain of patient 2 using Cartesian sampling pattern for WHT for different scan percentage with $\mathrm{TV}$ for $80,70,60,50,40$ percent sampling respectively

Figure 6.shows the reconstructed images at different sampling percentages for three types of MR images. The image quality is substantially good at $40 \%$ sampling retaining all the features as in original image. The proposed work can reconstruct MR images with considerably good quality with the lesser $\mathrm{k}$-space samples in comparison with the existing method which uses DFT as the sparsifying transform. Collecting lesser samples speeds up the MR process and hence the time of exposure of patient to the magnetic field reduces.

\section{CONCLUSION}

In the proposed work the MRI is reconstructed from the very few $\mathrm{k}$-space coefficients which are sparsified usingWalsh Hadamard Transform and the reconstructed image is compared with the standard method which uses DFT. The kspace is compressively sampled using Cartesian and using Radial patterns. For different sampling percentages the quality of the reconstructed MR image is analyzed in terms of PSNR, MSE and SSIM. The experimentation performed on different MR images demonstrates that the proposed method produces a faithful recovery even with lesser samples. The reconstruction with the proposed method is compared with the standard method which uses DFT, reveals that WHT can also be used as an effective sparsifying transform. Further the experimentation can be conducted with lesser sampling percentages. Smaller the number of $\mathrm{k}$-space samples the smaller will be the time the patient spends for the MR scanning process.

\section{REFERENCES}

1. Lustig M., Donoho D. and Pauly J.M., "Sparse MRI: The application of compressed sensing for rapid MR imaging", Magnetic Resonance in Medicine, vol. 58, pp. 1182-1195, 2007.

2. Kieren Grant Hollingsworth, "Reducing acquisition time in clinical MRI by data undersampling and compressed sensing reconstruction", Physics in Medicine \& Biology, vol. 60, pp. 297-322, 2015.

3. Donoho D., "Compressed sensing", IEEE Transaction on Information Theory, vol. 52(4), pp. 1289-1306, 2006.

4. Candès E.J and Wakin M.B., "An Introduction to Compressive Sampling”, IEEE Signal Processing Magazine, vol. 25(2), pp. 21-30, 2008.

5. Lustig M., Donoho D.L., Santos J.M. and Pauly J.M., "Compressed sensing MRI", IEEE Signal Processing Magazine, vol. 6, pp. 72-82, 2008.

6. Baker, Christopher Adams, "Investigation of Sparsifying Transforms in Compressed Sensing for Magnetic Resonance Imaging with Fasttestcs "The University of Wisconsin-Milwaukee Thesis and Dissertations, 2016.

7. Elizabeta S. Ilievska and Zoran A. Ivanovski, "Customized k-space trajectory for Compressed Sensing MRI", Proceedings of Telecommunications Forum vol. 23, pp.631-634, 2011.

8. Kai Tobias and Mainz, "Advanced Methods for Radial Data Sampling in Magnetic Resonance Imaging", ISMRM Workshop on Non-Cartesian MRI Sedona vol. 17, pp. 234-246, 2008.

9. Jing Liu and David Saloner, "Accelerated MRI with CIRcular Cartesian UnderSampling (CIRCUS): a variable density Cartesian sampling strategy for compressed sensing and parallel imaging”, Comprehensive Revision of Radiology, 2014.

10. JaganathanVellagoundar, M. Ramasubba Reddy, "Optimal k-space Sampling Scheme for Compressive Sensing MRI”, Proceedings of IEEE EMBS International Conference on Biomedical Engineering and Sciences, pp.531-534, 2012.

11. W.S. Besbas, Prof. M.A. Artemi, R.M. Suliman, M.M. AL_Rjebi, "Content Based Face Image Retrieval in WHT Domain”, vol. 86, pp.32-50,2008.

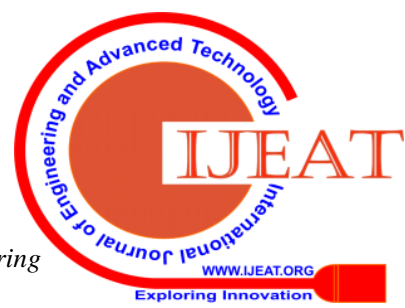


12. Shiqian Ma, Watao Yin and AmitChakraborty, "An Efficient Algorithm for Compressed MR Imaging using Total Variation and Wavelets", IEEE Conference on Computer Vision and Pattern Recognition, 2008.

13. Ravishankar S, Bresler Y. 'MR image reconstruction from highly under sampled k-space data by dictionary learning', IEEE Transaction on Medical Imaging vol. 30, pp.1028-41, 2010.

14. Shrividya G., Bharathi S.H., "A Study of Optimum Sampling Pattern for Reconstruction of MR images using Compressive Sensing", Proceedings of IEEE, 2nd International Conference on Advances in Electronics, Computers and Communication, Feb.2018.

15. Shrividya G. Bharathi S. H., "Application of Compressive Sensing on Magnetic Resonance Imaging: A brief survey", Proceedings of IEEE, International Conference on Recent Trends in Electronics, Information and Communication Technology, 2016. 